\title{
大陆深俯冲带变质流体活动过程中原岩锆石的物理搬运 和不活动性元素的化学迁移
}

\author{
盛英明 ${ }^{*}$, 郑永飞 \\ 中国科学院壳幔物质与环境重点实验室, 中国科学技术大学地球和空间科学学院, 合肥 230026 \\ *联系人, E-mail: ymsaint@ustc.edu.cn \\ 2013-06-18 收稿, 2013-06-26 接受, 2013-07-29 网络版发表 \\ 国家重点基础研究发展计划(2009CB825004)和国家自然科学基金(41221062, 40973004)资助
}

\begin{abstract}
摘要 对大别造山带超高压榴辉岩中不同类型的变质脉开展了变质铅石学和微区地球化学对 比研究, 包括矿物多相包裹体观察, 微量元素和氢氧同位素分析, 锆石内部结构和外部形态, $\mathrm{U}-\mathrm{Pb}$ 定年, 微量元素, Lu-Hf 和 $\mathrm{O}$ 同位素分析. 结果发现, 形成石英脉的流体是高压富水的, 引 起变质新生锆石的生长, 同时伴随原岩残留岩浆锆石的固态重结晶和溶解重结晶; 形成复杂矿 物组合脉的流体可能具有超临界性质, 引起原岩残留锆石的溶解, $\mathrm{Zr}$ 的短距离迁移以及变质新 生锆石再沉淀。两种不同类型的变质脉中残留岩浆铅石和变质新生锆石的同时存在，指示在俯 冲带变质流体活动过程中, 同时发生原岩残留岩浆锆石的物理搬运和元素 $\mathrm{Zr}$ 的化学迁移.
\end{abstract}

\section{关键词}

变质脉

流体活动

元素迁移

变质锆石学

大陆俯冲带
变质脱水和部分熔融是大陆碰撞造山带中两个 密切相关的过程 ${ }^{[1,2]}$, 对俯冲带变质温压条件下板块 界面的元素迁移行为具有重要研究意义 ${ }^{[3]}$. 与洋壳俯 冲带类似, 在进变质脱水过程中流体/熔体既可以与 一些元素迁移耦合, 也可以与另一些元素解耦. 这种 差异不仅取决于流体性质, 即富水流体、含水熔体或 者超临界流体 ${ }^{[4,5]}$, 而且取决于水和元素相对于地温 梯度和原岩性质的迁移程度和机制, 例如络合形式 和水/岩比 ${ }^{[3]}$. 俯冲带变质温压条件下湿固相线可能 终止于第二临界终点 ${ }^{[6-8]}$, 由此超临界流体形成并作 为强有力的传输介质 ${ }^{[2]}$. 由于含水熔体和超临界流体 中微量元素的溶解度显著增强, 因此它们在俯冲带 变质作用过程中是否出现, 在很大程度上决定了流 体活动和元素活动性的耦合和解耦关系 ${ }^{[2,6]}$.

大陆碰撞造山带变质岩中的脉体是变质流体发 生矿物沉淀的产物, 在超高压变质岩中很常见, 提供 了大陆俯冲带变质过程中流体活动的直接记录 ${ }^{[9 ~ 14]}$. 脉体的沉淀需要显著的变质流体流动, 这构成了潜
在的物质和能量迁移介质. 因此, 变质脉体见证了机 械变形和变质反应条件下流体和元素的再分配. 本 文概括了大别造山带超高压变质岩中两种类型淡色 脉体的岩石学和地球化学研究, 探讨了大陆深俯冲 带变质流体活动性质及其伴随的元素迁移行为.

\section{1 变质脉类型和成脉流体性质}

依据矿物共生组合, 可以将变质成因脉体简单 分为两大类, 一是富含石英的石英脉, 二是矿物组成 复杂的脉. 在石英脉的矿物组合中, 石英含量通常 $>95 \mathrm{wt} \%($ 图 1(a))，此外可含有副矿物蓝晶石、金红石、 钛铁矿、锆石、绿帘石、磷灰石和黄铁矿等 ${ }^{[9,13,15 \sim 18]}$. 在复杂变质脉中, 矿物组合变化极大, 寄主岩石类型 和成脉流体性质可能是其决定控制因素. 例如, 对于 大别造山带三祖寺超高压榴辉岩中的一条复杂变质 脉(图 1(b)), 其主要矿物为 20\%石英, $25 \%$ 蓝晶石, $15 \%$ 多硅白云母和 $25 \%$ 绿帘石, 副矿物为石榴石、金 红石、锆石和其他矿物 ${ }^{[14]}$. 


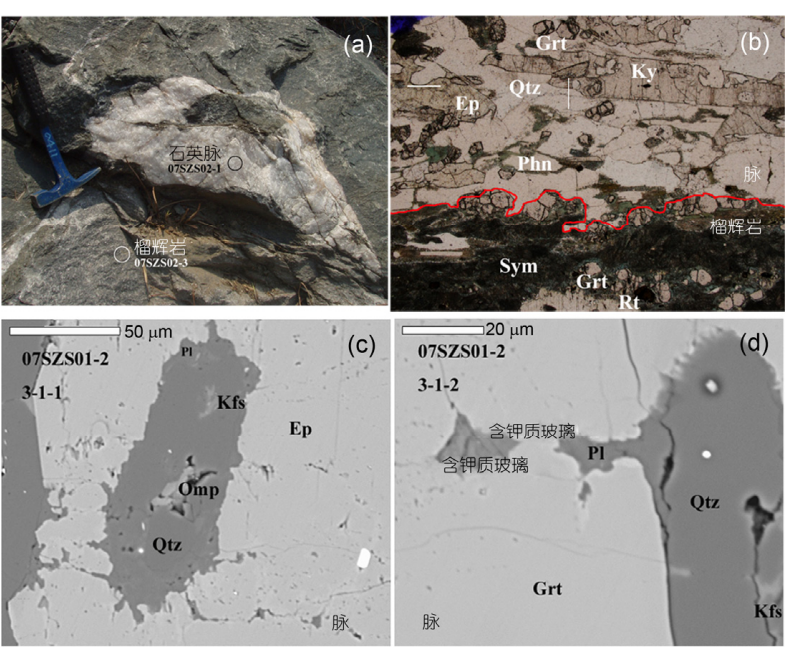

图 1 大别造山带两种不同类型变质脉的矿物共生组合及其 中多相固体包裹体

据 Sheng 等人 ${ }^{[13,14]}$ 修改. (a) 榴辉岩中石英脉野外产状, 主要构成矿 物为石英; (b) 显微镜下复杂变质脉的矿物共生组合, 主要矿物为 $20 \%$ 石英, $25 \%$ 蓝晶石, $15 \%$ 多硅白云母和 $25 \%$ 绿帘石; (c)和(d) 复杂 变质脉石榴石和绿帘石中石英土钾长石土斜长石土钾质玻璃原生多相 固体包裹体, 不规则或负晶形, 弱的放射状裂纹. Qtz, 石英; Grt, 石 榴石; Ep, 绿帘石; Phn, 多硅白云母; Ky, 蓝晶石; Rt, 金红石; Sym, 后成合晶; Kfs, 钾长石; Pl, 斜长石; Omp, 绿辉石

脉锆石中含有矿物和流体包裹体，其中石榴石、 绿辉石和石英等矿物与富水流体包裹体共存, 指示 石英脉形成于高压榴辉岩相 ${ }^{[17]}$, 成脉流体来源为深 俯冲大陆地壳折返过程中含水矿物分解以及名义上 无水矿物中结构差基和分子水出溶 ${ }^{[1]}$. 一方面高压条
件下富水流体的元素迁移能力十分有限，而另一方 面石英脉中出现流体不活动性元素载体矿物如石榴 石、金红石和锆石等, 说明 HREE 和 HFSE 发生了短 距离的迁移. 这是由于结构羟基释放产生的富水流 体是高度碱性的和氧化的，构成溶解和迁移 $\mathrm{Zr}$ (与 $\mathrm{OH}^{-}$形成络合物形式)和 $\mathrm{Si}$ 的有效传输介质 ${ }^{[9,19]}$. 在 石英脉矿物中没有发现柯石英, 结合锆石 U-Pb 定年 结果，指示石英脉形成于超高压变质岩的降压折返 阶段而非峰期超高压变质阶段 ${ }^{[9]}$.

在复杂矿物组合脉的石榴石和绿帘石中发现了 由石英士钾长石 \pm 斜长石 \pm 钾质玻璃组成的原生多相 固体包裹体(图 1(c), (d)), 表现为不规则或负晶形, 环绕弱的放射状裂纹，指示其形成于富 $\mathrm{Si}, \mathrm{Al}, \mathrm{K}$ 的流 体/熔体 ${ }^{[20 ~ 22]}$. 同时, 脉中显著富集含水矿物多硅白 云母和绿帘石, 表明成脉流体介质主要为富水流体 并包含少量含水熔体，在超高压条件下有可能成为 超临界流体 ${ }^{[2,5]}$. 在俯冲带变质条件下, 超临界流体 不仅能够迁移 LILE 和 LREE, 而且具备迁移 HREE 和 HFSE 的能力 ${ }^{[2,10,14,23]}$. Zhang 等人 ${ }^{[10]}$ 在这种复杂脉 体中发现柯石英, 指示其形成于超高压变质阶段.

\section{2 石英脉变质锆石学}

对大别造山带中温/超高压相带石英脉及其寄主 榴辉岩锆石进行了原位 SIMS 氧同位素和 U-Pb 年龄 分析(图 2,3), 以及 LA-(MC)-ICPMS 微量元素, U-Pb 和 Lu-Hf 同位素分析, 结果指示了大陆俯冲带条
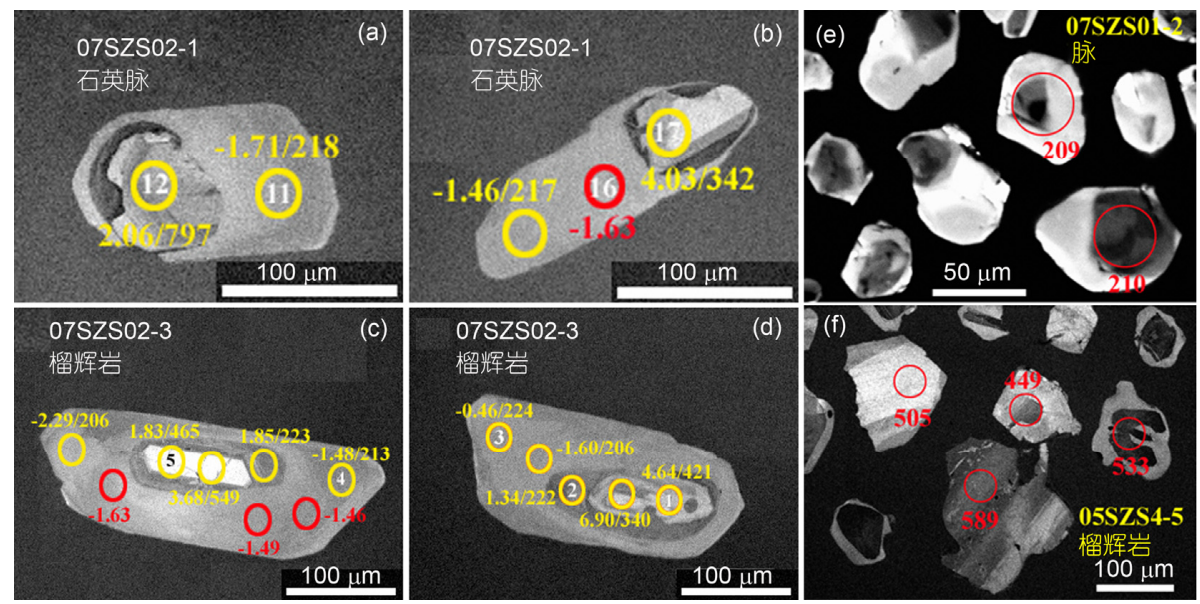

图 2 大别造山带变质脉及其寄主榴辉岩中锆石 CL 图像、氧同位素和 U-Pb 年龄

据 Sheng 等人 ${ }^{[13,14]}$ 修改. (a) (d) 石英脉及其寄主榴辉岩锆石, 脉锆石多呈长柱状, 并表现出核-(幔)-边结构, 核部为残留岩浆锆石发生固态重结 晶, 幔部为残留岩浆锆石发生溶解重结晶, 边部为变质新生, 黄色数字为 SIMS 原位测定的 $\delta^{18} \mathrm{O}$ 值 $(\% \circ) / \mathrm{U}-\mathrm{Pb}$ 年龄 $(\mathrm{Ma})$, 红色数字为 $\delta^{18} \mathrm{O}$ 值 $(\% o)$; (e)和(f) 复杂变质脉及其寄主榴辉岩锆石, 脉锆石多呈现浑圆状, 红色数字为 U-Pb 年龄 $(\mathrm{Ma})$ 
件下变质新生锆石和残留原岩锆石发生重结晶作用 的富水流体效应 ${ }^{[13,24]}$. 从锆石外部形态的对比中发 现 (图 2), 富水流体中变质生长锆石多表现为柱 状 ${ }^{[9,13,17,18]}$, 而含水熔体或超临界流体中变质生长锆 石多表现为不规则的浑圆状 ${ }^{[11,14,25]}$.

在 CL 图像上(图 2), 大多数锆石表现出明显的核(幔)-边结构, 边部无环带, 具有低的微量元素含量和 $\mathrm{Th} / \mathrm{U}$ 比值(0.003 0.06), 缓的 MREE-HREE 配分模型, 不明显的 $\mathrm{Eu}$ 负异常, 表明它们从变质流体中直接生

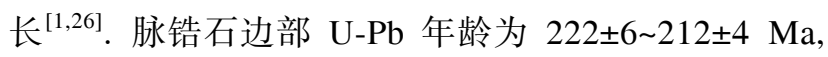
指示深俯冲大陆地壳折返过程中锆石的多期生长. 石英脉和榴辉岩中三叠纪 U-Pb 铅石边部具有相对均 一的负 $\delta^{18} \mathrm{O}$ 值(图 3), 其中石英脉为 $-2.59 \%$ - $1.23 \%$, 榴辉岩为 $-3.37 \%$ - $0.33 \%$, 表明它们从同一来源的 负 $\delta^{18} \mathrm{O}$ 变质流体中生长(图 3 中蓝色三角形).

石英脉和榴辉岩的锆石核部表现出清晰的振荡 环带或补丁状分区(图 2), 高的微量元素含量和 Th/U 比值(0.13 2.12), 陡峭的 MREE-HREE 配分模型, 显 著的 $\mathrm{Eu}$ 负异常, 新元古代上交点 U-Pb 年龄, 多数核 部具有正的 $\delta^{18} \mathrm{O}$ 值 (图 3 中黑色三角形), 指示其在富 水流体存在条件下的发生固态重结晶作用, U-Pb 和 O 同位素组成受到部分改造. 榴辉岩中少数锆石幔部

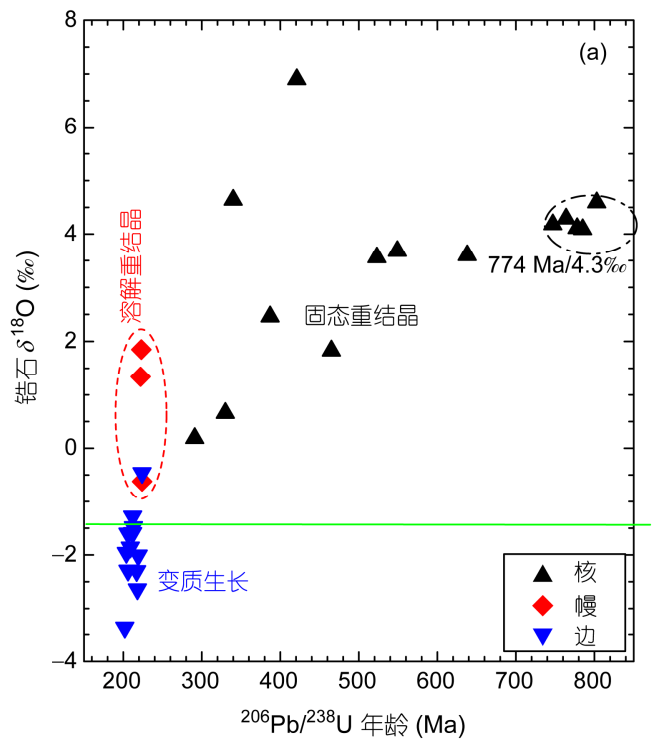

表现出正的 $\delta^{18} \mathrm{O}$ 值和三叠纪谐和 U-Pb 年龄(图 3(a) 中红色菱形), 表明这些原岩残留锆石在局部流体作 用下发生了溶解重结晶作用, $\mathrm{O}$ 同位素受到部分改造, 而 U-Pb 同位素被完全再造. 具有新元古代谐和 U-Pb 年龄的锆石核给出略低于正常地幔的 $\delta^{18} \mathrm{O}$ 值, 其中 榴辉岩为 $4.3 \%$, 石英脉为 $4.8 \%$ 。 这些残留岩浆锆石 和新生变质的锆石 $\mathrm{U}-\mathrm{Pb}$ 和 $\mathrm{O}$ 同位素特征与青龙山类 似 ${ }^{[24]}$, 即脱水流体较之原岩在 $\mathrm{O}$ 同位素比值上更低.

因此，锆石核部结晶自新元古代低 $\delta^{18} \mathrm{O}$ 岩浆, 锆石边部在三叠纪变质作用过程中从负 $\delta^{18} \mathrm{O}$ 富水流 体中直接生长. 石英脉中锆石核来自寄主榴辉岩, 是 被成脉流体所携带的捕虏晶. 脉中残留岩浆锆石核 与变质增生边的共存表明, 大陆碰撞造山带变质流 体同时具备锆石物理搬运和元素 $\mathrm{Zr}$ 的化学迁移能力. 在大陆俯冲带变质条件下, 原岩锆石在结晶程度上 的差异以及富水流体的活动性是引起原岩岩浆锆石 经历不同程度变质重结晶的基本控制因素 ${ }^{[1,25,27 ~ 29]}$.

\section{3 复杂矿物组合脉微量元素迁移和变质锆 石学}

对大别造山带榴辉岩中复杂矿物组合脉的详尽

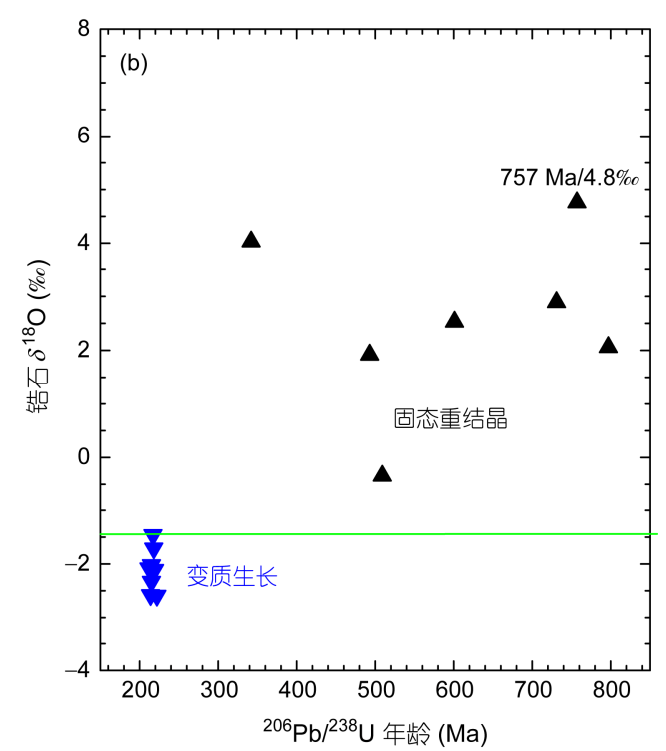

图 3 大别造山带变质脉及其寄主榴辉岩中锆石 U-Pb 年龄和 O 同位素相关性图解

据 Sheng 等人 ${ }^{[13] 1}$ 修改. (a) 榴辉岩锆石, 黑色三角代表新元古代低 $\delta^{18} \mathrm{O}$ 残留岩浆核, 正的 $\delta^{18} \mathrm{O}$ 值和早于三叠纪的 U-Pb 年龄, 指示在富水流体作 用下发生固态重结晶作用, U-Pb 和 $\mathrm{O}$ 同位素被部分改造; 蓝色三角代表变质新生边, 相对均一的 $\delta^{18} \mathrm{O}$ 值 $(-3 \%$ $-1 \%$ o 和三叠纪谐和 U-Pb 年龄, 表明从负 $\delta^{18} \mathrm{O}$ 富水流体中直接生长, 红色菱形代表残留岩浆锆石幔部, 正的 $\delta^{18} \mathrm{O}$ 值和三叠纪谐和年龄, 指示发生溶解重结晶作用, 氧同位素 被部分改造, 而 U-Pb 同位素被完全重置; (b) 石英脉锆石, 黑色和蓝色三角与榴辉岩锆石含义相同. 样品中新元古代谐和年龄锆石核被用来近 似指示初始岩浆 $\delta^{8} \mathrm{O}$ 值 

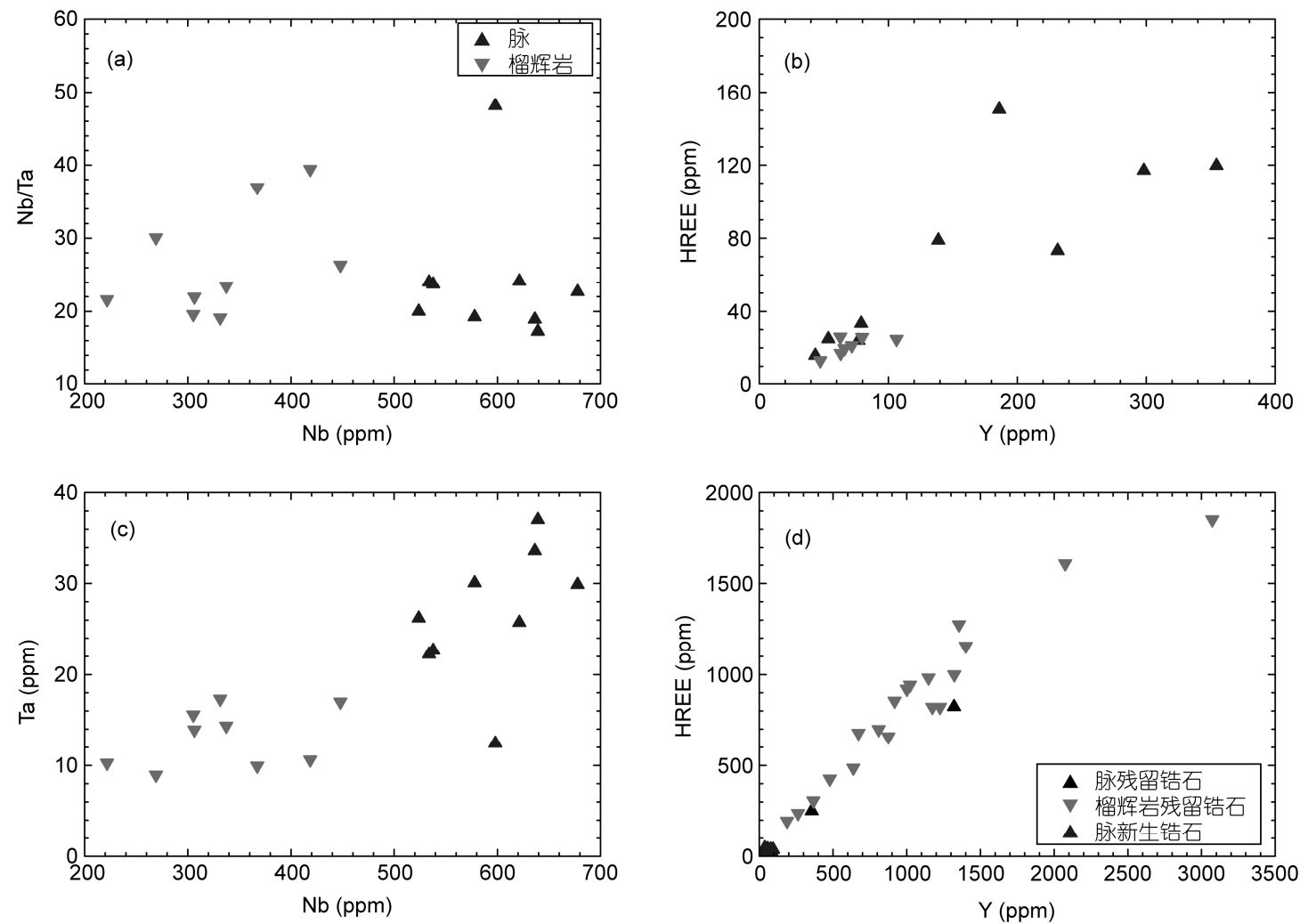

图 4 大别造山带复杂矿物组合脉元素活动性对比

据 Sheng 等人 ${ }^{[14]}$ 修改. (a)和(c) 变质脉和围岩榴辉岩中金红石 $\mathrm{Nb}$ 含量 $-\mathrm{Nb} / \mathrm{Ta}$ 比值和 $\mathrm{Nb}-\mathrm{Ta}$ 含量图解, 尽管二者 $\mathrm{Nb} / \mathrm{Ta}$ 比值无明显差异, 但是 脉金红石表现出较之榴辉岩明显高的 $\mathrm{Nb}$ 和 $\mathrm{Ta}$ 含量, 说明二者在不同变质阶段生长形成, 指示成脉流体作用下 $\mathrm{Nb}$ 和 $\mathrm{Ta}$ 在脉中的显著富集; (b) 脉中石榴石较之榴辉岩表现出高 HREE 和 $\mathrm{Y}$ 含量, 指示 HREE 和 $\mathrm{Y}$ 发生了明显的迁移; (d) 变质新生锆石全部发现于脉中, 指示了 $\mathrm{Zr}$ 和 $\mathrm{Hf}$ 同样发生显著的迁移和汇聚

的岩石学和地球化学研究 ${ }^{[14]}$. 结果发现, 复杂变质 脉较之榴辉岩含有大量多硅白云母和绿帘石，表明 流体活动性不相容微量元素 LILE 和 LREE 在成脉过 程中发生了显著的迁移和富集. 通过对矿物元素活 动性的对比(图 4)发现, 尽管脉和围岩中金红石 $\mathrm{Nb} / \mathrm{Ta}$ 比值没有明显差异，但是脉中金红石表现出较后者 明显高的 $\mathrm{Nb}$ 和 $\mathrm{Ta}$ 含量(1.8 2.0 倍), 说明二者形成于 不同变质阶段而非简单机械搬运，进而指示成脉流 体作用下 $\mathrm{Nb}$ 和 $\mathrm{Ta}$ 在脉中的显著迁移和富集. 脉中石 榴石较之榴辉岩中石榴石同样表现出高的 HREE 和 Y
含量(2.2 5.7 倍), 指示不活动性不相容微量元素 HREE 和 $\mathrm{Y}$ 亦发生明显的迁移. 变质新生锆石具有三叠纪 U$\mathrm{Pb}$ 年龄并且均出现在脉中, 脉与榴辉岩锆石表现出一 致的 $\mathrm{Hf}$ 同位素组成, $\varepsilon_{\mathrm{Hf}}(t)$ 分别为 $-3.3 \pm 1.2$ 和 $-3.6 \pm$ 2.0 - 3.1 \pm 1.4 , 指示不活动性元素 $\mathrm{Zr}$ 和 $\mathrm{Hf}$ 也发生了显 著的迁移和聚集. 因此, 在大陆俯冲带超高压变质条 件下, 岩石内部可能出现了部分熔融, 富水流体和含 水熔体可能具备了超临界性质, 结果这种流体不仅具 备流体活动性 LILE 和 LREE 的常见迁移能力, 而且具 备了流体不活动性 HREE 和 HFSE 的异常迁移能力.

\section{参考文献}

1 Zheng Y F. Fluid regime in continental subduction zones: Petrological insights from ultrahigh-pressure metamorphic rocks. J Geol Soc, 2009, 166: 763-782

2 Zheng Y F, Xia Q X, Chen R X, et al. Partial melting, fluid supercriticality and element mobility in ultrahigh-pressure metamorphic rocks during continental collision. Earth-Sci Rev, 2011, 107: 342-374 
3 Zheng Y F. Metamorphic chemical geodynamics in continental subduction zones. Chem Geol, 2012, 328: 5-48

4 Manning C. The chemistry of subduction-zone fluids. Earth Planet Sci Lett, 2004, 223: 1-16

5 Hermann J, Spandler C, Hack A, et al. Aqueous fluids and hydrous melts in high-pressure and ultrahigh pressure rocks: Implications for element transfer in subduction zones. Lithos, 2006, 92: 399-417

6 Kessel R, Schmidt M W, Ulmer P, et al. Trace element signature of subduction-zone fluids, melts and supercritical liquids at 120-180 km depth. Nature, 2005, 437: 724-727

7 Mibe K, Kawamoto T, Matsukage K N, et al. Slab melting versus slab dehydration in subductionzone magmatism. Proc Natl Acad Sci USA, 2011, 108: 8177-8182

8 Kawamoto T, Kanzaki M, Mibe K, et al. Separation of supercritical slab-fluids to form aqueous fluid and melt components in subduction zone magmatism. Proc Natl Acad Sci USA, 2012, 109: 18695-18700

9 Zheng Y F, Gao T S, Wu Y B, et al. Fluid flow during exhumation of deeply subducted continental crust: Zircon U-Pb age and O isotope studies of quartz vein in eclogite. J Metamorph Geol, 2007, 25: 267-283

10 Zhang Z M, Shen K, Sun W D, et al. Fluids in deeply subducted continental crust: Petrology, mineral chemistry and fluid inclusion of UHP metamorphic veins from the Sulu orogen, eastern China. Geochim Cosmochim Acta, 2008, 72: 3200-3228

11 Chen R X, Zheng Y F, Hu Z C. Episodic fluid action during exhumation of deeply subducted continental crust: Geochemical constraints from zoisite-quartz vein and host metabasite in the Dabie orogen. Lithos, 2012, 155: 146-166

12 Guo S, Ye K, Chen Y, et al. Fluid-rock interaction and element mobilization in UHP metabasalt: Constraints from an omphacite-epidote vein and host eclogites in the Dabie orogen. Lithos, 2012, 136-139: 145-167

13 Sheng Y M, Zheng Y F, Chen R X, et al. Fluid action on zircon growth and recrystallization during quartz veining within UHP eclogite: Insights from U-Pb ages, O-H isotopes and trace elements. Lithos, 2012, 136-139: 126-144

14 Sheng Y M, Zheng Y F, Li S N, et al. Element mobility during continental collision: Insights from polymineralic metamorphic vein within UHP eclogite in the Dabie orogen. J Metamorph Geol, 2013, 31: 221-241

15 Franz L, Romer R L, Klemd R, et al. Eclogite-facies quartz veins within metabasites of the Dabie Shan (eastern China): Pressuretemperature-time-deformation path, composition of the fluid phase and fluid flow during exhumation of high-pressure rocks. Contrib Mineral Petrol, 2001, 141: 322-346

16 Li X P, Zheng Y F, Wu Y B, et al. Low-T eclogite in the Dabie terrane of China: Petrological and isotopic constraints on fluid activity and radiometric dating. Contrib Mineral Petrol, 2004, 148: 443-470

$17 \mathrm{Wu} \mathrm{Y} \mathrm{B,} \mathrm{Gao} \mathrm{S,} \mathrm{Zhang} \mathrm{H} \mathrm{F,} \mathrm{et} \mathrm{al.} \mathrm{U-Pb} \mathrm{age,} \mathrm{trace-element,} \mathrm{and} \mathrm{Hf-isotope} \mathrm{compositions} \mathrm{of} \mathrm{zircon} \mathrm{in} \mathrm{a} \mathrm{quartz} \mathrm{vein} \mathrm{from} \mathrm{eclogite} \mathrm{in} \mathrm{the}$ western Dabie Mountains: Constraints on fluid flow during early exhumation of ultrahigh-pressure rocks. Am Miner, 2009, 94: 303-312

18 Zong K Q, Liu Y S, Hu Z C, et al. Melting-induced fluid flow during exhumation of gneisses of the Sulu ultrahigh-pressure terrane. Lithos, 2010, 120: 490-510

19 Ayers J C, Zhang L, Luo Y. Zircon solubility in alkaline aqueous fluids at upper crustal conditions. Geochim Cosmochim Acta, 2012, 96: 18-28

20 Zeng L S, Liang F H, Asimow P, et al. Partial melting of deeply subducted continental crust and the formation of quartzofeldspathic polyphase inclusions in the Sulu UHP eclogites. Chin Sci Bull, 2009, 54: 2580-2594

21 Gao X Y, Zheng Y F, Chen Y X. Dehydration melting of ultrahigh-pressure eclogite in the Dabie orogen: Evidence from multiphase solid inclusions in garnet. J Metamorph Geol, 2012, 30: 193-212

22 Chen Y X, Zheng Y F, Hu Z. Synexhumation anatexis of ultrahigh-pressure metamorphic rocks: Petrological evidence from granitic gneiss in the Sulu orogen. Lithos, 2013, 156-159: 69-96

$23 \mathrm{Xu} \mathrm{H} \mathrm{J,} \mathrm{Ye} \mathrm{K,} \mathrm{Song} \mathrm{Y,} \mathrm{et} \mathrm{al.} \mathrm{Prograde} \mathrm{metamorphism,} \mathrm{decompressional} \mathrm{partial} \mathrm{melting} \mathrm{and} \mathrm{subsequent} \mathrm{melt} \mathrm{fractional} \mathrm{crystallization} \mathrm{in}$ the Weihai migmatitic gneisses, Sulu UHP terrane, eastern China. Chem Geol, 2013, 341: 16-37

24 Chen Y X, Zheng Y F, Chen R X, et al. Metamorphic growth and recrystallization of zircons in extremely ${ }^{18} \mathrm{O}-\mathrm{depleted} \mathrm{rocks} \mathrm{during} \mathrm{eclogite-}$ facies metamorphism: Evidence from U-Pb ages, trace elements, and O-Hf isotopes. Geochim Cosmochim Acta, 2011, 75: 4877-4898

25 Xia Q X, Zheng Y F, Hu Z C. Trace elements in zircon and coexisting minerals from low-T/UHP metagranite in the Dabie orogen: Implications for action of supercritical fluid during continental subduction-zone metamorphism. Lithos, 2010, 114: 385-412

26 Rubatto D. Zircon trace element geochemistry: Partitioning with garnet and the link between U-Pb ages and metamorphism. Chem Geol, 2002, 184: 123-138

27 Xia Q X, Zheng Y F, Yuan H L, et al. Contrasting Lu-Hf and U-Th-Pb isotope systematics between metamorphic growth and recrystallization of zircon from eclogite-facies metagranites in the Dabie orogen, China. Lithos, 2009, 112: 477-496

28 Chen R X, Zheng Y F, Xie L W. Metamorphic growth and recrystallization of zircon: Distinction by simultaneous in-situ analyses of trace elements, U-Th- $\mathrm{Pb}$ and Lu-Hf isotopes in zircon from eclogite-facies rocks in the Sulu orogen. Lithos, 2010, 114: 132-154

29 Li W C, Chen R X, Zheng Y F, et al. Zirconological tracing of transition between aqueous fluid and hydrous melt in the crust: Constraints from pegmatite vein and host gneiss in the Sulu orogen. Lithos, 2013, 162-163: 157-174 